CGPG-96/9-5

gr-qc/9609048

\title{
The postulates of gravitational thermodynamics
}

\author{
Erik A. Martinez * \\ Center for Gravitational Physics and Geometry, \\ Department of Physics, \\ The Pennsylvania State University, \\ University Park, PA 16802-6300, USA
}

\begin{abstract}
The general principles and logical structure of a thermodynamic formalism that incorporates strongly self-gravitating systems are presented. This framework generalizes and simplifies the formulation of thermodynamics developed by Callen. The definition of extensive variables, the homogeneity properties of intensive parameters, and the fundamental problem of gravitational thermodynamics are discussed in detail. In particular, extensive parameters include quasilocal quantities and are naturally incorporated into a set of basic general postulates for thermodynamics. These include additivity of entropies (Massieu functions) and the generalized second law. Fundamental equations are no longer homogeneous first-order functions of their extensive variables. It is shown that the postulates lead to a formal resolution of the fundamental problem despite non-additivity of extensive parameters and thermodynamic potentials. Therefore, all the results of (gravitational) thermodynamics are an outgrowth of these postulates. The origin and nature of the differences with ordinary thermodynamics are analyzed. Consequences of the formalism include the (spatially) inhomogeneous character of thermodynamic equilibrium states, a reformulation of the Euler equation, and the absence of a GibbsDuhem relation.
\end{abstract}

PACS numbers: 04.70.Dy, 04.40.-b, 97.60.Lf

Typeset using REVTEX

*electronic address: martinez@phys.psu.edu 


\section{INTRODUCTION}

The inclusion of gravity into a physical theory fundamentally alters its basic assumptions and structure. Perhaps the most general and universal of all theories is thermodynamics. It is therefore particularly interesting to understand the changes induced by gravity in its underlying principles and to organize them into a general framework that serves as the foundation of a thermodynamic theory that incorporates strongly self-gravitating systems. Although this is relevant in its own right, it is also important in a somewhat different context: gravitational thermodynamics is expected to arise as one of the macroscopic limits of quantum gravity. Despite the fact that progress continues to be made into the way gravity alters the structure of quantum field theories [1], there does not exist yet a complete theory of quantum gravity. We believe that the characteristic features and principles of a theory of gravitational thermodynamics have to be understood properly before they can be fully

justified by means of statistical methods derived from one or several candidate theories of quantum gravity.

It is our purpose in this paper to formulate the general principles of gravitational thermodynamics. We shall discuss in detail the basic definitions and concepts of this formalism, its overall structure, its fundamental problem, the minimal set of assumptions (the postulates) that lead to the formal resolution of this problem as well as their mathematical and physical consequences. This formulation clarifies not only the differences and similarities between gravitational and nongravitational thermodynamics within a coherent framework, but also a number of existing misconceptions concerning its logical foundations and results. It generalizes the overall structure of thermodynamic theory and may provide a basic framework to incorporate current and future progress [1,2] in a statistical mechanical description of self-gravitating systems.

A clear and elegant formulation of the foundations and structure of ordinary (that is, nongravitational) thermodynamics has been introduced by Callen [3]. However, this formalism cannot describe the thermodynamics of gravitational systems in its present form and 
needs to be modified. To recognize this, we shall follow the following strategy. We believe that the most effective way to gain insight into the limitations of a set of physical principles is by studying a model system that illustrates them without unnecessary complications. We introduce such a model problem in Sec. II. It is the simplest example of a composite self-gravitating system at finite temperature and resembles as closely as possible the textbook example of a composite system in ordinary thermodynamics. The model is general enough to capture all the non-trivial behavior of gravity, but simple enough to allow an exact evaluation of all quantities, and provides insight into the places where the thermodynamic formalism has to be refined. As a preparatory step in the study of the postulatory basis of thermodynamics, we show that it provides a counterexample to a basic assertion [3, 4] of ordinary thermodynamics: in gravitational thermodynamics, additivity of entropies applied to spatially separate subsystems does not depend on or require the entropies of the latter to be homogeneous first-order functions of their extensive parameters.

The general principles of gravitational thermodynamics are developed from the start in Sec. III, which constitutes the core of the paper. It is shown that a general and rigorous characterization of the defining properties of thermodynamical self-gravitating systems originates from two factors, namely (1) the particular characteristics of their extensive variables, and (2) the homogeneous properties of their intensive parameters as functions of extensive variables. The correct definitions of these variables are discussed in detail. Extensive variables include quasilocally defined quantities like quasilocal energy and provide the background for the postulates. We discuss the composition of thermodynamic systems, the existence of fundamental equations, and formulate the fundamental problem of gravitational thermodynamics. Our object is to follow as closely as possible the logic of Callen's formalism and generalize its basic definitions and postulates wherever it is required. The definitions and postulates arrived at are a natural extension of the ones of ordinary thermodynamics with appropriate modifications necessary to accommodate the global aspects and nonlinearities characteristic of gravity. The postulates are the basic principles of thermodynamics when strongly self-gravitating systems are considered. We revise completely 
the logic of Sec. II and show that additivity of entropies (and, in general, additivity of all Massieu functions) as well as the generalized first and second laws of thermodynamics are part of these postulates and, as such, are not to be proven within the thermodynamic formalism. We demonstrate that the fundamental problem is formally solved by these postulates in spite of strong interaction between constituent subsystems and the associated nonadditivity of extensive variables. The preeminence of Massieu functions over thermodynamic potentials is discussed. The conditions of thermodynamical equilibrium and its (spatially) inhomogeneous nature (equivalence principle) are a direct result of the postulates.

Although the fundamental equation and the intensive variables maintain their mutual relationships, the former is no longer a homogeneous first-order function of extensive parameters. This property is not a consequence of the inhomogeneity of equilibrium configurations but of the functional form of the gravitational equations of state. These are in general no longer homogeneous zeroth-order functions under rescaling of extensive parameters. We show that these central differences with nongravitational thermodynamics are not forbidden by the logical structure of the formalism and can be incorporated easily into it by relaxing several assumptions in the postulates. However, the mathematical consequences of the new postulates are quite different from the ones familiar in regular thermodynamics. Formal relationships (like the Euler equation) must be reformulated and there is no direct analogue of the ordinary Gibbs-Duhem relation among intensive variables. Nonetheless, this does not influence significantly the applicability of the thermodynamic formalism.

The principles of the framework and its logic apply to general systems at finite temperature. They incorporate the so-called gravothermal catastrophe and other peculiar thermodynamical behavior observed in self-gravitating objects. As such, all standard equilibrium thermodynamics of gravitational configurations is a consequence of the general postulates presented here. The approach generalizes ordinary thermodynamics and provides a consistent treatment of composite self-gravitating systems (with or without matter fields). Moreover, it becomes evident that the modifications in the thermodynamic formalism necessary to incorporate gravity liberates it from assumptions that appeared (and are not) fundamen- 
tal, and as such, allows us to regard its general character and the extent of its logic in their full measure.

\section{HOMOGENEITY AND ADDITIVITY}

We motivate our presentation of the postulates of gravitational thermodynamics and their consequences with a model problem. It consists of a spherically symmetric uncharged black hole surrounded by matter (represented by a thin shell). We shall find in this section the relationship among the entropy functions $S_{0}, S_{B}$, and $S_{S}$ whenever thermodynamic equilibrium is achieved and its connection with the functional dependence of those functions. In what follows, subscripts $B$ and $S$ refer to quantities for black hole and shell constituents respectively, whereas the subscript 0 refers to quantities for the total system.

The composite system is characterized by the surface area $A_{0}=4 \pi r_{0}^{2}$ of a two-dimensional spherical boundary surface $B_{0}$ (located at $r=r_{0}$ ) that encloses the components, and the quasilocal energy $E_{0}$ contained within [56]. The Arnowitt-Deser-Misner (ADM) mass $m_{+}$ of the system as a function of these variables is

$$
m_{+}\left(E_{0}, A_{0}\right)=E_{0}\left(1-\frac{E_{0}}{2 r_{0}}\right) .
$$

Throughout the paper, units are chosen so that $G=\hbar=c=k_{\text {Boltzmann }}=1$. Due to spherical symmetry of the model problem, we use areas and radii interchangeably. The pressure $p_{0}$ associated to the surface $B_{0}$ is obtained as (minus) the partial derivative of the energy $E_{0}$ with respect to $A_{0}$ at constant $m_{+}$. It corresponds to a negative surface tension and reads

$$
p_{0}\left(E_{0}, A_{0}\right)=\frac{E_{0}{ }^{2}}{16 \pi r_{0}{ }^{3}}\left(1-\frac{E_{0}}{r_{0}}\right)^{-1}=\frac{1}{16 \pi r_{0} k_{0}}\left(1-k_{0}\right)^{2},
$$

where $k_{0} \equiv\left(1-2 m_{+} / r_{0}\right)^{1 / 2}$. Let $\beta_{0}$ denote the inverse temperature at the surface $B_{0}$. The first law of thermodynamics for the system reads

$$
d S_{0}=\beta_{0}\left(d E_{0}+p_{0} d A_{0}\right),
$$


which can be written as a total differential by using Eqs. 2.1) and (2.2) as

$$
d S_{0}=\beta_{0} k_{0}^{-1} d m_{+}
$$

Consider now the constituent systems. The black hole is characterized by the surface area $A_{B}=4 \pi R^{2}$ of a spherical boundary surface $B_{R}$ located at $r=R \leq r_{0}$, and by its quasilocal energy $E_{B}$. The ADM mass $m_{-}$of the black hole as a function of these variables is 6 ]

$$
m_{-}\left(E_{B}, A_{B}\right)=E_{B}\left(1-\frac{E_{B}}{2 R}\right)
$$

The horizon radius of the black hole is $2 m_{-}$. The different radii satisfy the inequalities $2 m_{-} \leq 2 m_{+} \leq R \leq r_{0}$, where $2 m_{+}$represents the horizon radius of the total system. The pressure associated to the gravitational field of the black hole at the surface $B_{R}$ is

$$
p_{B}\left(E_{B}, A_{B}\right)=\frac{E_{B}{ }^{2}}{16 \pi R^{3}}\left(1-\frac{E_{B}}{R}\right)^{-1}=\frac{1}{16 \pi R k_{-}}\left(1-k_{-}\right)^{2},
$$

where $k_{-} \equiv\left(1-2 m_{-} / R\right)^{1 / 2}$. As it is well known, the first law of thermodynamics for the black hole can be expressed as a total differential by using Eqs. (2.5) and (2.6), namely

$$
\begin{aligned}
d S_{B} & =\beta_{B}\left(d E_{B}+p_{B} d A_{B}\right) \\
& =\beta_{B} k_{-}^{-1} d m_{-}
\end{aligned}
$$

where $\beta_{B}$ is the inverse temperature of the black hole at the surface $B_{R}$. This equation must be contrasted with the entropy differential (2.4) for the total system.

For thermodynamical purposes, the shell is considered (effectively) at rest. For simplicity, we assume that the shell surface coincides with the surface $B_{R}$. (This does not imply loss of generality [5].) Thus, the surface areas for the black hole and shell coincide: $A_{B}=A_{S} \equiv$ $A_{R}=4 \pi R^{2}$. The gravitational junction conditions [7,8] at the shell position determine its surface energy density $\sigma$ and surface pressure $p_{S}$ to be

$$
E_{S} \equiv 4 \pi R^{2} \sigma=R\left(k_{-}-k_{+}\right)
$$


and

$$
p_{S}=\frac{1}{16 \pi R k_{-} k_{+}}\left[k_{-}\left(1-k_{+}\right)^{2}-k_{+}\left(1-k_{-}\right)^{2}\right]
$$

respectively. The symbol $E_{S}$ denotes the local mass-energy of matter and $k_{+} \equiv(1-$ $\left.2 m_{+} / R\right)^{1 / 2}$. To simplify the analysis further, we consider only the case when the total number of particles $N_{S}$ in the shell is constant. The condition $m_{+} \geq m_{-}$guarantees that both $E_{S}$ and $p_{S}$ are non-negative. Let $\beta_{S}$ denote the inverse local temperature at the shell. Its entropy differential reads

$$
d S_{S}=\beta_{S}\left(d E_{S}+p_{S} d A_{R}\right)
$$

Use of Eqs. (2.8) and (2.9) allows us to write the matter entropy as

$$
d S_{S}=\beta_{S}\left(k_{+}^{-1} d m_{+}-k_{-}^{-1} d m_{-}\right) .
$$

How are the three entropies related when the system is in equilibrium? The emergence of equilibrium conditions from general principles within thermodynamics is the subject of the following section. However, intuitively the system is in a state of thermal equilibrium provided (1) $\beta_{B}=\beta_{S} \equiv \beta_{R}$, and (2) $\beta_{R}=\beta_{0} k_{0}{ }^{-1} k_{+}$at the surface $B_{R}$. The first condition constrains the temperature at the shell surface to coincide with the black hole temperature there (black hole and shell in mutual thermal equilibrium), whereas the second guarantees that the total system is in thermal equilibrium with its components 9]. Mechanical equilibrium of the matter shell with the black hole is guaranteed by the shell pressure equation (2.9). Under these conditions, Eqs. (2.4), (2.7), and (2.10) jointly imply that

$$
d S_{0}=d S_{B}+d S_{S}
$$

The entropy of the composite system is, therefore, additive with respect to its constituent subsystems. Since the entropy is a function of energy and size (its "extensive variables" discussed below) this means that, up to a global constant,

$$
S_{0}\left(E_{0}, A_{0}\right)=S_{B}\left(E_{B}, A_{R}\right)+S_{S}\left(E_{S}, A_{R}\right)
$$


In the preceding analysis, additivity is a direct consequence of the conditions of thermal and mechanical equilibrium. Observe that it is independent of the functional form of the parameters $\beta_{S}\left(E_{S}, A_{R}\right)$ and $\beta_{B}\left(E_{B}, A_{R}\right)$. This is as expected, since inverse temperature appears in the first law of thermodynamics as an integrating factor. In particular, the foregoing derivation of additivity does not depend on the special choice of boundary conditions or phenomenological matter action employed in Ref. [5] or on spherical symmetry [10]. It depends only on the adopted definition of stress-energy tensor [11] in terms of quasilocal quantities.

As discussed in the following section, the entropies $S_{0}\left(E_{0}, A_{0}\right), S_{B}\left(E_{B}, A_{R}\right)$, and $S_{S}\left(E_{S}, A_{R}\right)$ can be determined from Eqs. (2.4), (2.7) and (2.10) only if the precise forms of all the thermodynamical equations of state are known. The latter express intensive parameters as functions of the appropriate extensive parameters. For example, it is well known that if the thermal equation of state for a black hole is given by Hawking's semiclassical expression [12]

$$
\beta_{B}\left(E_{B}, A_{R}\right)=8 \pi E_{B}\left(1-\frac{E_{B}}{2 R}\right)\left(1-\frac{E_{B}}{R}\right),
$$

then Eq. (2.7) yields the Bekenstein-Hawking entropy [6]

$$
S_{B}\left(E_{B}, A_{R}\right)=4 \pi E_{B}{ }^{2}\left(1-\frac{E_{B}}{2 R}\right)^{2}=4 \pi m_{-}{ }^{2} .
$$

These expressions are sufficient to demonstrate that additivity of entropies for spatially separate subsystems does not require the entropy of each constituent system to be a homogeneous first-order function of its extensive parameters. This is in contrast to ordinary thermodynamics [3: [4]. Recall that a function $f\left(x_{1}, \ldots, x_{n}\right)$ is said to be a homogeneous $m$-th order function of the variables $\left(x_{1}, \ldots, x_{n}\right)$ if it satisfies the identity

$$
f\left(\lambda x_{1}, \ldots, \lambda x_{n}\right)=\lambda^{m} f\left(x_{1}, \ldots, x_{n}\right),
$$

where $\lambda$ is a constant. Upon the rescaling $E_{B} \rightarrow \lambda E_{B}, A_{R} \rightarrow \lambda^{2} A_{R}(R \rightarrow \lambda R)$ the entropy $S_{B}\left(E_{B}, A_{R}\right)$ in Eq. (2.15) behaves as a homogeneous second-order function of $E_{B}$ and as a first-order function of $A_{R}$, namely [6,5] 


$$
S_{B}\left(\lambda E_{B}, \lambda^{2} A_{R}\right)=\lambda^{2} S_{B}\left(E_{B}, A_{R}\right) .
$$

Equations (2.14) and (2.6) also illustrate a central characteristic of gravitational systems: the inverse temperature and pressure are not homogeneous zeroth-order functions. Un-

der rescaling they behave as [6] $\beta_{B}\left(\lambda E_{B}, \lambda^{2} A_{R}\right)=\lambda \beta_{B}\left(E_{B}, A_{R}\right)$ and $p_{B}\left(\lambda E_{B}, \lambda^{2} A_{R}\right)=$ $\lambda^{-1} p_{B}\left(E_{B}, A_{R}\right)$.

The functional form of $S_{S}\left(E_{S}, A_{R}\right)$ and its homogeneous properties depend on the explicit form of the matter equations of state. These arise from either a phenomenological or a field theoretical description of the matter fields involved, and their precise form does not concern gravity. Examples of equations of state for a self-gravitating matter shell (in the absence of a black hole) in thermal equilibrium with itself have been studied in Ref. [13]. Observe that Eq. (2.2) for $p_{0}$ and Eq. (2.6) for $p_{B}$ are indeed equations of state while Eq. (2.9) for $p_{S}$ is not. If the equations of state were known for both components, the total entropy $S_{0}$ could be obtained by Eq. (2.13) as a function of the extensive parameters of the subsystems. A discussion of this point and of further properties of this model are delayed to the following section.

\section{THE FUNDAMENTAL PROBLEM AND THE POSTULATES}

The preceding analysis motivates the search for principles that are independent of model problems and that incorporate the characteristics of gravity into a logical framework more general than ordinary thermodynamics. We must start, therefore, by reviewing the basic assumptions.

Gravitational thermodynamics describes (effectively) static states of macroscopic finitesize self-gravitating systems. As in regular thermodynamics, it is expected that very few variables survive the statistical average involved in a macroscopic measurement. What are these macroscopic parameters? The relationship between thermodynamical and dynamical variables in gravity has been amply discussed and we refer the reader to the literature [14 [17]. For our purposes, it is enough to recall the following points. Firstly, it has been shown in 
a wide variety of problems (involving black holes at finite temperature in interaction with matter fields) that the thermodynamical energy coincides with the quasilocal energy $E$ that naturally follows from the action of a spatially bounded region. If ${ }^{3} B$ denotes the threedimensional boundary of the system and ${ }^{2} B$ the two-surface resulting from its intersection with a spacelike hypersurface $\Sigma$, the quasilocal energy is the value of the Hamiltonian that generates unit time translations on ${ }^{3} B$ in the direction orthogonal to the surface ${ }^{2} B$ [14, 11]. We postulate that this is the appropriate energy variable in (gravitational) thermodynamics for all self-gravitating systems. Secondly, the analog of the thermodynamical "size" of the system is the induced two-metric $\sigma_{a b}$ of the two-dimensional boundary surface ${ }^{2} B$ [6, 18, 14]. This property reflects the "surface character" of gravitational thermodynamics and is in part a consequence of the lack of an operational definition of "volume" in the presence of black holes. The size reduces to the surface area $A$ of the two-surface only in the case of spherical symmetry [14]. For composite systems, quantities that measure size for internal matter components have to be found. Finally, the remaining macroscopic variables are a finite number of conserved charges. These may include, for example, angular momentum [14], suitable combinations of electric [19] or magnetic [20] charges, cosmological constant [21], other types of hair [22], and number of particles for matter systems [13]. (The thermodynamic conjugate quantities to these parameters are chemical potentials defined at the boundary of the system $19,14$.

The existence of these macroscopic parameters motivates the first postulate:

Postulate I: There exist particular states (called equilibrium states) of self-gravitating systems that are completely characterized macroscopically by the specification of a finite set of variables. These variables are the quasilocal energy, size, and a small number of conserved quantities (denoted generically by the symbol $N$ ).

In ordinary thermodynamics a similar postulate is usually applied to so-called "simple" systems [3]. These systems do not include gravitational or electromagnetic fields and are by definition macroscopically homogeneous. The previous postulate incorporates strongly selfgravitating configurations (with or without matter fields). As shown below, these systems 
may be spatially inhomogeneous. Systems describable by these parameters may be termed "simple" in gravitational thermodynamics.

Observe that the preceding postulate does not imply that every gravitational system has equilibrium configurations. Very often a system does not possess an equilibrium state even though it has definite values of energy and other parameters. Rather, the postulate maintains that equilibrium states, in case they exist, are completely described by the foregoing finite set of parameters.

The variables $\left(E, \sigma_{a b}, N\right)$ that describe a gravitational equilibrium state are to be called extensive parameters. Extensive quantities are constructed entirely from the dynamical phase space variables. Another essential difference with usual thermodynamics appears here: in the latter, the extensive parameters of a composite system equal the sum of their values in each of the subsystems. As we illustrate below, this is not the case in gravitational thermodynamics.

Some extensive variables of a self-gravitating system cannot be constrained in the conventional thermodynamic sense. For example, there exist no walls restrictive with respect to angular momentum of a stationary black hole system. However, this is not unusual or particular to gravity. For instance, it also occurs in the treatment of magnetic systems: there exist no walls restrictive with respect to magnetic moment. However, one can maintain always the value of magnetic moment constant at a boundary surface that delimits the system by a feedback mechanism that continually adjusts the magnetic field [3]. The same happens in gravitational thermodynamics: unconstrainable quantities can be kept constant at a given boundary surface by continually monitoring the value of their respective conjugate chemical potentials at this surface. The unavailability of walls that restrict certain extensive variables is only an idiosyncrasy that does not affect the applicability of thermodynamics. As in ordinary thermodynamics, a boundary that does (does not) allow the flux of heat can be called diathermal (adiabatic). Observe that the quasilocal energy adopted here has a very important property for thermodynamics. It is "macroscopically controllable" in the usual thermodynamic sense: it can be "trapped" by restrictive boundaries and "manipu- 
lated" by diathermal ones. A boundary that does not allow the flow of heat and work can be called "restrictive with respect to quasilocal energy." A closed system is defined as one whose extensive variables (quasilocal energy, size, etc.) remain effectively constant at its boundary surface.

It is of course difficult to split a self-gravitating system into independent "component" systems in the manner familiar in ordinary thermodynamics. Although one can speak of a "composite" system formed by two or more subsystems, the latter interact strongly among themselves. Clearly, if a composite system is closed, the simple systems are not necessarily so. However, internal constraints may exist among the component systems. These are constraints that prevent the flow of energy or any other extensive parameter among subsystems. For example, in our model problem internal constraints can restrict the flow of energy between the two subsystems (for instance, by fixing a particular value of $E_{B}$ ) or area (by fixing $\left.A_{R}\right)$. The relaxation of internal constraints in an equilibrium composite system will create processes that will tend to bring the system to a new equilibrium state.

The central problem of thermodynamics of strongly self-gravitating systems is, therefore, a reflection of the central problem of ordinary thermodynamics [3], namely: The determination of the equilibrium states that will result when internal constraints are removed in a closed, composite system.

What assumptions are needed in order to solve this problem? Equilibrium states in gravitational thermodynamics must be characterized by a simple extremum principle. As any other thermodynamic system, a gravitational system will select, in the absence of constraints, any one of a number of states, each of which can also be realized in the presence of a suitable constraint. Each of these constrained equilibrium states corresponds to particular values of the extensive parameters of each constituent system and has a definite entropy. Therefore, the extremum postulate states that if constraints are lifted, the system will select the state with the largest entropy. Paraphrasing Callen: 
Postulate II: There exists a single-valued function (the entropy $S$ ) of the extensive variables of any composite system, defined for all equilibrium states, and possessing the following property. In the absence of internal constraints, the values assumed by the extensive parameters are those that maximize the entropy over the manifold of constrained equilibrium states.

This postulate has to be interpreted carefully. Physical equilibrium states correspond to states that extremize the total entropy over the manifold of constrained equilibrium states. Equilibrium states are, therefore, either maxima, minima, or inflection points of the entropy. However, in the absence of constraints, the extensive parameters of the components in the final equilibrium state will be those that maximize the entropy. Postulates I and II not only predict equilibrium states, but also determine their stability properties. Equilibrium states corresponding to maxima of entropy are stable whereas unstable equilibrium states correspond to extrema other than maxima. It is important to emphasize that Postulate II makes no reference to nonequilibrium states. Furthermore, it implies neither that all equilibrium states of a gravitational system must have maximum entropy nor that stable states do exist. After all, it is common to find systems possessing equilibrium states that are local minima of entropy. Simple examples include a nonrelativistic self-gravitating gas in a spherical box or isothermal stellar systems [23].

The entropy as a function of its extensive variables constitutes the "fundamental equation" of a self-gravitating system. The first differentials of the fundamental equation define its intensive variables. For systems with a vanishing shift vector, the intensive variables in the entropy representation are $(\beta, \beta p, \beta \mu)$, where $\beta$ denotes inverse temperature, $p$ pressure, and $\mu$ chemical potentials. Systems possessing a nonvanishing shift require functional differentials in the definitions of their intensive parameters. (This happens, for example, in the thermodynamic description of stationary geometries [14.) For general spacetimes the conjugate quantities to the size $\sigma_{a b}$ are proportional to (minus) the spatial stresses introduced in Ref. [11]. The intensive parameters are always functions of the extensive parameters. The set of functional relationships expressing intensive in terms of extensive parameters are the 
thermodynamical equations of state of a self-gravitating system. For example, for static (as opposed to stationary) systems these are

$$
\begin{gathered}
\beta=\beta(E, A, N), \\
\beta p=\beta p(E, A, N), \\
\beta \mu=\beta \mu(E, A, N) .
\end{gathered}
$$

As in ordinary thermodynamics, once the fundamental equation $S\left(E, \sigma_{a b}, N\right)$ of a system (or, alternatively, its complete set of equations of state) is known, all its thermodynamical information can be obtained from it.

The criteria for global and local stability of equilibrium states in terms of the entropy function are identical to the ones familiar in ordinary thermodynamics [3, 13]. In particular, global stability requires that the entropy hypersurface $S\left(E, \sigma_{a b}, N\right)$ lies everywhere below its family of tangent hyperplanes.

It is possible to express the fundamental equation in terms of different sets of independent variables by performing Legendre transformations on the entropy. These are the so-called Massieu functions [24].3]. They play a more fundamental role in gravitational than in ordinary thermodynamics because they are in a one-to-one correspondence with actions [15]. Their preeminence over "thermodynamic potentials" has not been emphasized sufficiently. (The latter are Legendre transforms of energy and include the Helmholtz potential $F$ and Gibbs potential $G$.) For static systems, Massieu functions include, for example, $\mathcal{S}_{1}(\beta, A, N)=S-\beta E=-\beta F$, in which quasilocal energy has been replaced by its conjugate entropic intensive parameter (inverse temperature) as independent variable, and $\mathcal{S}_{2}(\beta, \beta p, N)=S-\beta E-\beta p A=-\beta G$ in which, in addition, the size of the system is replaced by its entropic intensive parameter. The above equations can be generalized to stationary geometries if one recalls that in general it is not possible to choose all intensive variables constant at a given choice of two-dimensional boundary surface [14,25]. The basic extremum postulate is very general and can be reformulated in these alternative representations: each Legendre transform of the entropy is a maximum for constant values of the transformed 
(intensive) parameters [3].

It is important to emphasize that the second postulate incorporates not only the so-called first law, but also the generalized second law [26 28] into a thermodynamic formalism.

Finally, how is the entropy of a composite system related to the entropies of the subsystems? In the previous section we illustrated that entropies are additive despite strong interaction between subsystems. On the one hand, additivity of entropies does not seem to depend critically on the particular functional form of the intensive parameters. On the other hand, it seems to be a natural consequence of the additivity of actions in a path integral approach to statistical thermodynamics [5, 10]. These reasons motivate us to assume the additivity postulate:

Postulate III: The entropy of a composite system is additive over the constituent subsystems. Furthermore, it is a continuous, differentiable, and monotonically increasing function of the quasilocal energy $E$.

We emphasize three important points. First, we shall not assume in this postulate that the entropy of each subsystem is a homogeneous first-order function of the extensive parameters. The postulate is, therefore, more general than the corresponding one in regular thermodynamics [3], 1$]$. Second, the preceding postulate implies that all Massieu functions are additive over component Massieu functions. As we illustrate below, this is not the case for thermodynamic potentials [5]. Third, the monotonic property implies that the temperature is postulated to be non-negative as in ordinary thermodynamics.

The logic of the previous section must be contrasted with the present one: additivity is neither the result of equilibrium conditions among intensive variables, nor of the functional form of intensive or extensive parameters, but a fundamental assumption. Additivity is valid even when the subsystems cannot be considered independent and strongly interact among themselves. As we show in the following paragraphs, equilibrium conditions are indeed a consequence of the postulates.

The preceding postulates are the natural extension of the postulates of nongravitational thermodynamics necessary to accommodate the extensive parameters characteristic of grav- 
itational systems. Are these postulates sufficient to solve the fundamental problem despite strong interactions among subsystems? The answer is on the affirmative. To illustrate this consider again our model problem in the light of the logic resulting from the postulates. We shall determine the equilibrium state of the closed, composite system, namely, the relationships that must exist among extensive variables of the subsystems for the total system to be in thermal and mechanical (and in general, chemical) equilibrium. We also shall indicate how far one can proceed in the explicit solution of this problem without assuming particular expressions for the equations of state of the subsystems.

By Postulate I, the black hole and matter subsystems are simple systems characterized by the extensive variables $\left(E_{B}, A_{B}, N_{B}\right)$ and $\left(E_{S}, A_{S}, N_{S}\right)$, respectively. The composite system is itself a simple system and is characterized by the variables $\left(E_{0}, A_{0}, N_{0}\right)$. The size of all systems reduces to the area of their respective surfaces. By Postulate II, the fundamental thermodynamical equations in the entropy representation are the functions $S_{0}=S_{0}\left(E_{0}, A_{0}, N_{0}\right), S_{B}=S_{B}\left(E_{B}, A_{B}, N_{B}\right)$, and $S_{S}=S_{S}\left(E_{S}, A_{S}, N_{S}\right)$. Postulate III states that $S_{0}\left(E_{0}, A_{0}, N_{0}\right)=S_{B}\left(E_{B}, A_{B}, N_{B}\right)+S_{S}\left(E_{S}, A_{S}, N_{S}\right)$. For simplicity and with no loss of generality, we assume $A_{B}=A_{S} \equiv A_{R}$ and the quantities $N_{0}, N_{B}$ and $N_{S}$ to be constant. The system is considered closed if its quasilocal energy and area are kept effectively constant at the boundary $B_{0}$, namely

$$
E_{0}=\text { const.; } A_{0}=\text { const. }
$$

The fundamental problem is to determine the extensive variables $\left(E_{B}, E_{S}, A_{R}\right)$ as functions of these constant quantities whenever equilibrium is attained as a result of relaxing internal constraints. Postulate II establishes that the total entropy of a composite system in a state of equilibrium is an extremum, namely, it does not change as a result of an infinitesimal virtual transfer of heat or work from one subsystem to the other. Therefore, in equilibrium

$$
\begin{aligned}
d S_{0}=0 & =d S_{B}+d S_{S} \\
& =\beta_{B}\left(d E_{B}+p_{B} d A_{R}\right)+\beta_{S}\left(d E_{S}+p_{S} d A_{R}\right),
\end{aligned}
$$


where the second equality is a consequence of Postulates I and II. The entropic intensive variables are defined in the conventional way:

$\beta_{S}\left(E_{S}, A_{R}\right) \equiv\left(\partial S_{S} / \partial E_{S}\right)_{A_{R}}, \beta_{S} p_{S}\left(E_{S}, A_{R}\right) \equiv\left(\partial S_{S} / \partial A_{R}\right)_{E_{S}} ; \beta_{B}\left(E_{B}, A_{R}\right) \equiv\left(\partial S_{B} / \partial E_{B}\right)_{A_{R}}$, and $\beta_{B} p_{B}\left(E_{B}, A_{R}\right) \equiv\left(\partial S_{B} / \partial A_{R}\right)_{E_{B}}$.

Since the quasilocal energy can be expressed as $E_{0}=r_{0}\left(1-k_{0}\right)$, the closure equations are equivalent to the condition $m_{+}=$const. Because the energy $E_{B}$ refers to the surface $B_{R}$ which coincides with the shell surface, it is easy to see that the total quasilocal energy at $B_{R}$ is

$$
\begin{aligned}
E_{R} & \equiv E_{B}+E_{S} \\
& =R\left(1-k_{+}\right) .
\end{aligned}
$$

This equation is a consequence of the additivity of quasilocal energy discussed in [5, 11]. (If the black hole energy $E_{B}$ is defined at a surface which does not coincide with the shell surface, the energies $E_{B}$ and $E_{S}$ are not simply additive as in Eq. (3.4) [0].) The closure conditions and Eq. (3.4) allow the total entropy (3.3) to be written as

$$
d S_{0}=0=\left(\beta_{S}-\beta_{B}\right) d E_{S}+\left(\beta_{B} p_{B}+\beta_{S} p_{S}-\beta_{B} p_{R}\right) d A_{R}
$$

where the pressure $p_{R}$ is defined as

$$
p_{R}\left(E_{R}, A_{R}\right) \equiv \frac{E_{R}^{2}}{16 \pi R^{3}}\left(1-\frac{E_{R}}{R}\right)^{-1}=\frac{1}{16 \pi R k_{+}}\left(1-k_{+}\right)^{2} .
$$

Since the equality in Eq. (3.5) must be satisfied by independent and arbitrary variations of $E_{S}$ and $A_{R}$, we must necessarily have

$$
\beta_{S}=\beta_{B} \equiv \beta_{R}
$$

and

$$
p_{S}+p_{B}=p_{R}
$$

The preceding equations are the sought equilibrium conditions. They state the relationship among intensive variables of the subsystems for the composite system to be in thermal 
and mechanical equilibrium. As in nongravitational thermodynamics, they yield a formal solution to the fundamental problem provided the equations of state

$$
\begin{aligned}
& \beta_{B}=\beta_{B}\left(E_{B}, A_{R}, N_{B}\right), p_{B}=p_{B}\left(E_{B}, A_{R}, N_{B}\right) ; \\
& \beta_{S}=\beta_{S}\left(E_{S}, A_{R}, N_{S}\right), p_{S}=p_{S}\left(E_{S}, A_{R}, N_{S}\right)
\end{aligned}
$$

for the subsystems are known. If this is so, Eqs. (3.7) and (3.8) are two formal relationships among $E_{B}, E_{S}, A_{R}$ and $m_{+}$(with $N_{S}$ and $N_{B}$ each held fixed). Equations (3.2), (3.4), (3.7), and (3.8) are, therefore, the four desired equations that determine the four sought variables $\left(E_{B}, E_{S}, A_{R}, m_{+}\right)$.

Naturally, the variable $m_{+}$in Eqs. (3.2) and (3.4) does not play an important role in the formalism: the relationship among the three energies $E_{0}, E_{B}$, and $E_{S}$ can be written explicitly as

$$
E_{0}=r_{0}\left\{1-\left[1-\frac{2\left(E_{B}+E_{S}\right)}{r_{0}}\left(1-\frac{E_{B}+E_{S}}{2 R}\right)\right]^{1 / 2}\right\} .
$$

For a closed system, Eqs. (3.7), (3.8), and (3.10) (with Eq. (3.2)) provide three desired equations to determine the three sought variables $\left(E_{B}, E_{S}, A_{R}\right)$.

The fundamental problem is formally solved by the postulates despite the quasilocal energy $E_{0}$ not being the simple sum of the component energies $E_{B}$ and $E_{S}$ due to binding and self-energy interactions characteristic of gravitational systems. (It is easy to see, by using Eq. (3.10) that all thermodynamic potentials obtained from $E_{0}$ by Legendre transforms are not the simple sum of the component potentials.) This indicates not only that the postulates form a complete set of assumptions for a more general class of thermodynamic systems than previously considered, but also the appropriateness of the adopted definitions of extensive parameters.

Consider some further consequences of the postulates. Firstly, the mechanical equilibrium condition (3.8) represents the spatial stress component of the junction conditions at the surface $B_{R}$. (It reduces to Eq. (2.9) if the pressure equation of state for the black hole is given by (2.6)). Secondly, additivity of entropies and the equilibrium conditions (3.7) and (3.8) allow the differential of the total entropy to be written as 


$$
\begin{aligned}
d S_{0} & =\beta_{0}\left(d E_{0}+p_{0} d A_{0}\right) \\
& =\beta_{R}\left(d\left(E_{B}+E_{S}\right)+\left(p_{B}+p_{S}\right) d A_{R}\right) \\
& =\beta_{R}\left(d E_{R}+p_{R} d A_{R}\right) .
\end{aligned}
$$

This expression confirms that there is no "gravitational" entropy associated to the shell [29,5] and illustrates that $E_{R}$ is the total quasilocal energy and $p_{R}$ the associated pressure associated to the surface $B_{R}$. In turn, Eq. (3.11) implies that

$$
d S_{0}=\beta_{0} k_{0}^{-1} d m_{+}=\beta_{R} k_{+}^{-1} d m_{+} .
$$

Therefore, the inverse temperature at the surface $B_{R}$ is given in terms of the inverse temperature $\beta_{0}$ at the boundary $B_{0}$ by

$$
\beta_{0}{k_{0}}^{-1}=\beta_{R} k_{+}^{-1}
$$

The (spatially) inhomogeneous character [9] of thermodynamic equilibrium (equivalence principle) is a consequence of the postulates of thermodynamics and the definition of quasilocal stress-energy. Thus, the postulates do incorporate equilibrium states in inhomogeneous systems in contrast to the ordinary postulates of thermodynamics [30], where a system that is not homogeneous is not in thermodynamic equilibrium even if its properties remain constant in time.

The preceding treatment of a composite self-gravitating system must be contrasted with the equivalent one of a composite nongravitational system presented in Appendix A. Although the systems are physically different, their similarities and differences are readily apparent. In particular, the gravitational equations (3.10) and (3.2) substitute the relations (A1) and (A2) of flat spacetime thermodynamics.

The formalism provides the methodology to solve the fundamental problem for selfgravitating systems. In the spirit of thermodynamics, it yields explicit answers for explicit functional forms of the fundamental equations (or equivalently, the associated equations of state) of each of the subsystems [3]. These are outside the realm of thermodynamics and are 
the result of either phenomenological or statistical mechanical descriptions of the constituent systems. We reiterate its formal structure: for a composite self-gravitating system one must assume the fundamental equation of the components to be known in principle. If the total system is in a constrained equilibrium state (characterized by particular values of the extensive parameters for each constituent system), the total entropy is obtained by adding the individual entropies of the components and is, therefore, a known function of their extensive parameters. The extrema of the total entropy define the equilibrium states. Stable states correspond to maxima of entropy. As an illustration, if explicit equations of state are known for both black hole and matter in our model problem, their entropy values can be found (up to an overall constant) by integrating Eqs. (2.7) and (2.10), and substituting the values of $\left(E_{B}, E_{S}, A_{R}\right)$ at equilibrium. The total entropy is then given by Eq. (2.13).

Can this logical framework accommodate runaway instabilities (the so-called gravothermal catastrophe) observed in bounded self-gravitating systems? The answer is on the affirmative. This behavior is a consequence of the postulates and the particular form of the fundamental equations characteristic of gravity. Typically, the latter are such that there exist, besides equilibrium states that locally maximize the entropy, equilibrium states that locally minimize it. (The existence of these state is well-known in stellar dynamics [23] and black hole physics [6,18].) Consider as illustration an isothermal self-gravitating gas in a closed spherical container [23]. The system might be thought of as formed by two components, the 'core' and the 'halo.' The formalism states that if the entropies for the components are known as functions of their extensive parameters, the total entropy is $S=S_{c}+S_{h}$. Equilibrium states are obtained by extremizing this function and are characterized by particular values of the extensive parameters of the components. For simplicity, consider only the energies (or equivalently, the density contrast between components). The entropy functions for the gas components are such that there exists an equilibrium state (described by a particular critical value of the density contrast) that is a local entropy minimum over the set of all possible equilibrium states [23]. But Postulate II predicts that this state is unstable. The 
onset of instability in the gas obeys the postulates: if the system finds itself in that state and the density contrast between core and halo is allowed to change, the system will try to reach equilibrium states of higher entropy. The system finds it advantageous to transfer energy (or work) from one region to another, developing more internal inhomogeneities. Local stability conditions [3,13] imply that a negative heat capacity is associated to a local entropy minimum: if the core gets hotter than the halo, heat flows from the core to the halo and the core temperature raises. The end result depends on the form of the entropy function and on the direction of the fluctuation that started the instability. It might be that a local entropy maximum exists in which the system settles down (as discussed in Ref. [23], this occurs if the entropy is such that, for example, $C_{h}<\left|C_{c}\right|$ ). In this case the halo temperature rises more than the core's and the system shuts off in a stable state. A runaway instability happens if there does not exist a local maximum for the system to settle down (this happens if the fundamental equation is such that $C_{h}>\left|C_{c}\right|$ ). In this case the temperature difference between halo and core keeps growing. Whether a black hole is created or the system runs out of equilibrium before that occurs, the important point is that, as long as the system can be described by equilibrium physics, the postulates predict its behavior if the fundamental equations of the components are known. The above argument applies equally to a collection of stars or other astronomical systems.

We have studied so far the impact of gravitational extensive variables in the thermodynamic formalism. But the latter is also characterized by the functional forms of its intensive variables (equations of state). These arise from a dynamical theory but their main characteristic is that, in general, they are no longer homogeneous zeroth-order functions of extensive parameters (for instance, the inverse temperature $\beta_{B}$ in Eq. (2.14) is homogeneous first-order in energy and half-order in area; although the intensive variable $\beta p$ in the entropy representation of a static black hole is homogeneous zeroth-order, this is not the case for other systems [13].) This implies that the consequences of the postulates are different than in ordinary thermodynamics, particularly the mathematical properties of fundamental equations. Fundamental equations are in general no longer homogeneous first-order functions of their 
extensive variables. (Alternatively, the homogeneous properties of fundamental equations in gravitational thermodynamics imply that intensive variables are no longer homogeneous zeroth-order functions.) This does not affect the formalism itself, but has direct implications for at least two formal relationships among thermodynamic quantities. Firstly, the so-called Euler relation is necessarily different from the one familiar in ordinary thermodynamics [6]. An Euler relation is a consequence of Euler theorem stating that a homogeneous function $f\left(x_{1}, \ldots, x_{n}\right)$ of $m$-th order satisfies the equality

$$
m f\left(x_{1}, \ldots, x_{n}\right)=x_{1}\left(\frac{\partial f}{\partial x_{1}}\right)+\ldots+x_{n}\left(\frac{\partial f}{\partial x_{n}}\right) .
$$

In standard thermodynamics entropy is a homogeneous first-order function and the Euler relation is therefore $S=\beta E+\beta p V-\beta \mu N$. In contrast and as an example, the Euler relation for a static charged black hole reads

$$
S=\frac{1}{2} \beta E+\beta p A-\frac{1}{2} \beta \mu N
$$

Euler relations for hollow self-gravitating thin shells with power law thermal equations of state have been presented in Ref. [13].

Secondly, there does not exist a Gibbs-Duhem relation in gravitational thermodynamics [13]. The Gibbs-Duhem relation in ordinary thermodynamics is a direct consequence of the homogeneous first-order properties of the fundamental equation and relates the intensive parameters of a system. It states that the sum of products of extensive parameters and the differentials of the (conjugate) intensive parameters vanishes. In the entropy representation it reads $E d \beta+V d(\beta p)-N d(\beta \mu)=0$. In contrast, if one combines the first law with the Euler relation (3.15) for a charged black hole one obtains

$$
E d \beta-\beta d E+2 A d(\beta p)-N d(\beta \mu)+\beta \mu d N=0
$$

The reformulation of an Euler relation and the absence of a Gibbs-Duhem relation set gravity apart from other interactions: even for magnetic systems the Euler and the Gibbs-Duhem expressions maintain their usual relationship. 
There are no obstacles in applying the preceding formalism to any self-gravitating system. These may include not only nonrelativistic astronomical objects, but also highly relativistic systems involving general black holes in interaction with matter. The (spherically symmetric) model problem was used only as an illustration because of its simplicity and transparency. For general situations, a larger state space is required to incorporate a larger number of extensive variables and thermodynamic equilibrium includes equilibrium under "interchange of number $N$." It is possible to employ a condensed notation where the symbols $X_{i}$ and $P_{j}$ denote generically all extensive and intensive parameters, respectively (excluding energy and inverse temperature), as in Ref. [3]. In this way the equilibrium conditions (3.7) and (3.8) are easily generalized to include all chemical potentials for the system. Although the treatment of these and other systems may be technically difficult, the resolution of the fundamental problem of thermodynamics obeys the same logical structure as the one presented here.

We emphasize again that the characteristics of gravitational thermodynamics are the result of its extensive parameters (which must include quasilocal quantities like quasilocal energy) and the particular homogeneity properties of its intensive variables (equations of state) as functions of extensive parameters. This is a more general and rigorous characterization of the defining properties of gravitational thermodynamics than, for example, the one presented in Ref. 31] in the context of black holes. The definitions of extensive and intensive variables as well as the changes introduced in Callen's postulates are the minimal changes necessary to incorporate the global aspects and nonlinearities characteristic of the gravitational interaction into a postulatory formulation of thermodynamics.

To summarize, we have presented the overall structure and principles of a thermodynamic framework that incorporates self-gravitating systems. All the results of standard (gravitational) thermodynamics are a consequence of the generalized postulates and the solution of the fundamental problem and can be extracted from them by following the standard procedures described in Refs. [3. 32]. Possible applications of the formalism include, for example, the description of quasi-static reversible and irreversible processes, alternative representa- 
tions, phase transitions, etc. Finally, a further postulate is usually introduced in standard thermodynamics: the so-called third law. However, the main body of thermodynamics does not require this postulate since in the latter there is no meaning for the absolute value (and therefore for the zero) of entropy. The role and interpretation of this kind of assumption in the statistical mechanics of gravitation is the subject of a future publication [33].

\section{ACKNOWLEDGMENTS}

It is a pleasure to thank Abhay Ashtekar, Valeri Frolov, Gerald Horwitz, Werner Israel, Lee Smolin and especially James York for stimulating conversations and critical remarks. Research support was received from the National Science Foundation Grants No. PHY 9396246 and No. PHY 95-14240, and from the Eberly Research Funds of The Pennsylvania State University.

\section{APPENDIX A:}

The simplest closed, composite system in ordinary thermodynamics consists of two subsystems separated by a movable diathermal wall [3,30]. The fundamental problem is to determine the extensive variables $\left(E_{1}, V_{1}, N_{1}\right)$ and $\left(E_{2}, V_{2}, N_{2}\right)$ of the subsystems whenever equilibrium is attained. The quantities $E, V$, and $N$ refer to internal energy, volume, and number of particles, respectively. The closure conditions are

$$
\begin{aligned}
& E_{T} \equiv E_{1}+E_{2}=\text { const. } \\
& V_{T} \equiv V_{1}+V_{2}=\text { const. } \\
& N_{T} \equiv N_{1}+N_{2}=\text { const. }
\end{aligned}
$$

The postulates of thermodynamics allow a formal solution of the problem. The conditions of equilibrium follow from the equation

$$
d S_{T}=0=d S_{1}+d S_{2}
$$




$$
\begin{aligned}
& =\beta_{1}\left(d E_{1}+p_{1} d V_{1}-\mu_{1} d N_{1}\right)+\beta_{2}\left(d E_{2}+p_{2} d V_{2}-\mu_{2} d N_{2}\right) \\
& =\left(\beta_{1}-\beta_{2}\right) d E_{1}+\left(\beta_{1} p_{1}-\beta_{2} p_{2}\right) d V_{1}-\left(\beta_{1} \mu_{1}-\beta_{2} \mu_{2}\right) d N_{1} .
\end{aligned}
$$

The first equality is a consequence of the extremum value of entropy (Postulate II) whereas the second is a consequence of additivity of entropies (Postulate III). The last equality follows from the closure conditions. Since the variations $d E_{1}, d V_{1}$, and $d N_{1}$ are independent, Eq. (A4) implies that in the entropy representation

$$
\begin{gathered}
\beta_{1}=\beta_{2}, \\
\beta_{1} p_{1}=\beta_{2} p_{2}, \\
\beta_{1} \mu_{1}=\beta_{2} \mu_{2} .
\end{gathered}
$$

If the equations of state

$$
\begin{aligned}
& \beta_{a}=\beta_{a}\left(E_{a}, V_{a}, N_{a}\right), \\
& p_{a}=p_{a}\left(E_{a}, V_{a}, N_{a}\right), \\
& \mu_{a}=\mu_{a}\left(E_{a}, V_{a}, N_{a}\right)
\end{aligned}
$$

are known for the subsystems (with $a=1,2$ ), then Eqs. (A5), (A6), and (A7) provide three formal relationships among $\left(E_{1}, V_{1}, N_{1}\right)$ and $\left(E_{2}, V_{2}, N_{2}\right)$. Eqs. (A1), (A2), and (A3) together with Eqs. (A5), (A6) and (A7) are, therefore, the six desired equations necessary to determine the six unknown variables. 


\section{REFERENCES}

[1] See, for example, A. Ashtekar and J. Lewandowski, "Quantum field theory of geometry", preprint, CGPG-96/3-2, hep-th/9603083, and references cited therein.

[2] For some recent approaches, see G. Horowitz, "The origin of black hole entropy in string theory", preprint, UCSBTH-96-07, gr-qc/9604051; C. Rovelli, "Black hole entropy from loop quantum gravity", preprint, gr-qc/9603063.

[3] H. B. Callen, Thermodynamics and an Introduction to Thermostatistics, (Wiley, New York, 1985).

[4] R. F. Greene and H. B. Callen, Phys. Rev. 83, 1231 (1951).

[5] E. A. Martinez and J. W. York, Jr., Phys. Rev. D 40, 2124 (1989).

[6] J. W. York, Jr., Phys. Rev. D 33, 2092 (1986).

[7] W. Israel, Nuovo Cimento 44B, 1 (1966); 48B, 463 (1967); V. de la Cruz and W. Israel, Nuovo Cimento 51A, 774 (1967).

[8] C. Lanczos, Phys. Z. 23, 539 (1922); Ann. Phys. (Leipzig) 74, 518 (1924).

[9] R. C. Tolman, Relativity, Thermodynamics, and Cosmology, (Oxford University Press, Oxford, 1935).

[10] O. B. Zaslavskii, Phys. Lett. A 152, 463 (1991).

[11] J. D. Brown and J. W. York, Jr., Phys. Rev. D 47, 1407 (1993).

[12] S. W. Hawking, Commun. Math. Phys. 43, 199 (1975).

[13] E. A. Martinez, Phys. Rev. D 53, 7062 (1996).

[14] J. D. Brown, E. A. Martinez, and J. W. York, Jr., Phys. Rev. Lett., 66, 2281 (1991); in Nonlinear Problems in Relativity and Cosmology, edited by J. R. Buchler, S. L. Detweiler, and J.R. Ipser (New York Academy of Sciences, New York, 1991). 
[15] J. D. Brown, G. L. Comer, E. A. Martinez, J. Melmed, B. F. Whiting, and J. W. York, Jr., Class. Quantum Grav. 7, 1433 (1990).

[16] J. D. Brown and J. W. York, Jr., in Quantum Mechanics of Fundamental Systems 4, The Black Hole Twenty Five Years After, edited by C. Teitelboim and J. Zanelli (Plenum, New York, in press).

[17] J. Louko and B. F. Whiting, Phys. Rev. D 51, 5583 (1995).

[18] B. F. Whiting and J. W. York, Jr., Phys. Rev. Lett. 61, 1336 (1988).

[19] H. Braden, J. D. Brown, B. F. Whiting, and J. W. York, Jr., Phys. Rev. D 42, 3376 (1990); O. B. Zaslavskii, Phys. Rev. Lett. 76, 2211 (1996).

[20] O. B. Zaslavskii, Class. Quantum Grav. 12, L63 (1995).

[21] J. D. Brown, J. Creighton, and R. B. Mann, Phys. Rev. D 50, 6394 (1994).

[22] See, for example, D. Nunez, H. Quevedo, and D. Sudarsky, Phys. Rev. Lett. 76, 571 (1996), and references cited therein.

[23] J. Binney and S. Tremaine, Galactic Dynamics (Princeton University Press, Princeton, NJ, 1987).

[24] M. J. Massieu, C. R. Acad. Sci., Paris 69, 858 (1869).

[25] J. D. Brown and J. W. York, Jr., Phys. Rev. D 47, 1420 (1993).

[26] J. D. Bekenstein, Phys. Rev. D 23, 287 (1981).

[27] V. P. Frolov and D. N. Page, Phys. Rev. Lett. 71, 3902 (1993).

[28] O. B. Zaslavskii, Class. Quantum Grav. 13, L7 (1996).

[29] P. C. W. Davies, L. H. Ford, and D. N. Page, Phys. Rev. D 34, 1700 (1986).

[30] G. Carrington, Basic Thermodynamics (Oxford University Press, New York, 1994). 
[31] V. P. Frolov, Phys. Rev. Lett. 74, 3319 (1995).

[32] E. A. Guggenheim, Thermodynamics (North Holland, Amsterdam, 1967).

[33] E. A. Martinez (in preparation). 